\title{
Morphology Determines Conductivity and Seebeck Coefficient in Conjugated Polymer Blends
}

Guangzheng Zuo, Xianjie Liu, Mats Fahlman and Martijn Kemerink

The self-archived postprint version of this journal article is available at Linköping University Institutional Repository (DiVA):

http:/ / urn.kb.se/ resolve?urn=urn:nbn:se:liu:diva-147581

N.B.: When citing this work, cite the original publication.

Zuo, G., Liu, X., Fahlman, M., Kemerink, M., (2018), Morphology Determines Conductivity and Seebeck Coefficient in Conjugated Polymer Blends, ACS Applied Materials and Interfaces, 10(11), 9638-9644. https:// doi.org/ 10.1021/ acsami.8b00122

Original publication available at:

https:/ / doi.org/ 10.1021/ acsami.8b00122

Copyright: American Chemical Society

http:// pubs.acs.org/ 


\title{
Morphology Determines Conductivity and Seebeck Coefficient in Conjugated Polymer Blends
}

\author{
Guangzheng Zuo ${ }^{a}$, Xianjie Liu ${ }^{b}$, Mats Fahlman ${ }^{b}$ and Martijn Kemerink ${ }^{a}$ * \\ ${ }^{a}$ Complex Materials and Devices, Department of Physics, Chemistry and Biology, Linköping \\ University, 58183, Linköping, Sweden \\ ${ }^{\mathrm{b}}$ Division of Surface Physics and Chemistry, Department of Physics, Chemistry and Biology, \\ Linköping University, 58183 Linköping, Sweden
}

Keywords: Seebeck coefficient; morphology; charge transport; conjugated polymers; kinetic Monto Carlo simulations

Abstract

The impact of nanoscale morphology on conductivity and Seebeck coefficient in p-type doped all-polymer blend systems is investigated. For a strongly phase separated system (P3HT:PTB7), we achieve a Seebeck coefficient that peaks at $S \sim 1100 \mu \mathrm{V} / \mathrm{K}$ with conductivity $\sigma \sim 3 \cdot 10^{-3} \mathrm{~S} / \mathrm{cm}$ for 90\% PTB7. In marked contrast, for well mixed systems (P3HT:PTB7 with 5\% DIO, P3HT:PCPDTBT), we find an almost constant $S \sim 140 \mu \mathrm{V} / \mathrm{K}$ and $\sigma \sim 1 \mathrm{~S} / \mathrm{cm}$ despite the energy levels being (virtually) identical in both cases. The results are interpreted in terms of a variable 
range hopping (VRH) model where a peak in $S$ and a minimum in $\sigma$ arise when the percolation pathway contains both host and guest sites, in which the latter acts as energetic trap. For wellmixed blends of the investigated compositions, VRH enables percolation pathways that only involve isolated guest sites, whereas the large distance between guest clusters in phase separated blends enforces (energetically unfavorable) hops via the host. The experimentally observed trends are in good agreement with the results of atomistic kinetic Monte Carlo simulations accounting for the differences in nanoscale morphology. 


\section{Introduction}

Morphology heavily impacts the performance of organic electronic devices, especially in blends of disordered organic semiconductors (OSC), and consequently this is a hot research focus at present. For instance, in organic solar cells processing additives and the choice of solvent/cosolvent and drying time have been varied to reach optimized blend morphologies ${ }^{1-3}$ Kouijzer et al. predicted the morphology evolution of solution processed polymers-fullerene blends while going from liquid to film ${ }^{4}$, and the resulting phase separation with/without co-solvent in polymers-fullerene blends. ${ }^{5}$ In some cases, it is possible to qualitatively predict performance in relation to morphology, for example, by assuming the length scale of phase separation in photovoltaic blends gives an optimized PCE when it is about equal to the exciton diffusion length. ${ }^{6}$ In general, however, quantitative understanding of the relation between morphology and performance is still difficult.

It has recently become clear that morphology is -unsurprisingly- also a crucial factor when optimizing the thermoelectric properties of doped OSC. For example, Patel et al. revealed that the power factor in a doped OSC depends strongly on long-range ordering, and achieved a PF around $120 \mu \mathrm{W} / \mathrm{m} \cdot \mathrm{K}^{-2}$ by a morphology-preserving (vapor-based) doping process of the organic semiconductor. ${ }^{7}$ Hamidi-Sakr et al. showed the influence of morphology on anisotropic thermoelectric properties and charge transport in a family of $\mathrm{F}_{4}$ TCNQ-doped single polythiophene derivatives with different alkyl side chains. ${ }^{8}$ Also in two-component blends (or three-component if one counts the dopant) like PEDOT derivatives, various types of processing techniques can be used to strongly influence the system's conductivity $\sigma$, Seebeck coefficient $S$, thermal conductivity $\kappa$, or all. ${ }^{9-11}$ While it is known that the investigated processing techniques change the phase separation and ordering of the blend's constituents, the effects on the power 
factor $P F=S^{2} \sigma$, and thereby on the thermoelectric figure of merit $Z T=P F / \kappa T$, are at best partially understood. ${ }^{12-14}$ The reported highest PF is over $300 \mu \mathrm{W} / \mathrm{m} \cdot \mathrm{K}^{-2}$ and ZT reaches 0.25 in a PEDOT-Tos system. ${ }^{15}$ Recently, Saxena et al. showed that an improvement of thermoelectric properties of PEDOT:PSS can be achieved by the introduction of silicon nanoparticles that simultaneously affect conductivity, thermopower and thermal conductivity. ${ }^{16} \mathrm{Lu}$ et al. demonstrated that the thermoelectric properties of P3BT blended with insulating polystyrene compared to that of pure P3BT were improved by controlling the blend morphology to form an interpenetrating network. ${ }^{17}$ Otherwise, the insulating material in semiconductor/insulator blends mainly affects the conductivity via dilution effects while having a negligible impact on the Seebeck coefficient. ${ }^{18-19}$ In a previous work we showed that judicious blending of two OSC with different energy levels can be used to design the density of states such that it leads to very high thermopowers (>> $1000 \mu \mathrm{V} / \mathrm{K}$ ) at reasonable conductivities, but the role of morphology was not addressed. ${ }^{19}$ Summarizing, while it is evident that multi-component blends of organic (semi)conductors and insulators offer unique opportunities for achieving high PF and ZT, there is still a limited understanding of the impact of morphology on the thermoelectric performance of these systems.

Here, we experimentally and numerically investigate the conductivity and Seebeck coefficient

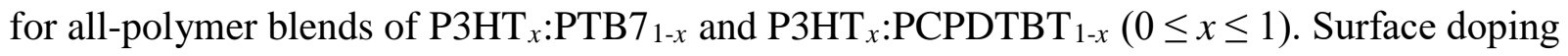
by $\mathrm{F}_{4} \mathrm{TCNQ}$ is used to enhance conductivity without distorting the film morphology. ${ }^{20}$ While the energy levels in both blends are virtually identical, AFM images showed that the P3HT:PTB7 system inclined to phase separation whereas the P3HT:PCPDTBT system mixed well for all compositions. For the $\mathrm{P} 3 \mathrm{HT}_{x}$ :PTB7 1 - system we found that the thermopower peaked at $S \sim 1100$ $\mu \mathrm{V} / \mathrm{K}$ for $x=0.9$. In stark contrast, $S$ was almost constant at $S \sim 150 \mu \mathrm{V} / \mathrm{K}$ for the 
P3HT:PCPDTBT system. When 5\% DIO was added into $\mathrm{P} \mathrm{HT}_{0.1}: \mathrm{PTB}_{0.9}$ to suppress the phase separation, the Seebeck coefficient dropped to $S \sim 400 \mu \mathrm{V} / \mathrm{K}$. We demonstrate that the observed changes in Seebeck coefficient and conductivity can directly be correlated to the morphology by assuming that charge and energy transport in our devices occurs by a variable range hopping (VRH) process. An important and general consequence of this is that in well-mixed binary blends, electron (hole) transport is dominated by the compound with the lowest LUMO (highest HOMO), even if this ‘trapping' compound makes up only $~ 5-10 \%$ of all sites. A kinetic Monto Carlo model that is based on VRH is able to reproduce the key features of our experiments.

\section{Results and discussion}

\subsection{Energy levels and morphology}

We chose two conjugated polymers with nominally the same energy levels, PTB7 and PCPDTBT, and blended these with P3HT, covering the full binary composition range. The ionization potential (IP) and Fermi energy of the three CPs were investigated by ultraviolet photoemission spectroscopy (UPS). The UPS spectra and the extracted IP and $E_{F}$ values are shown as Figure S2 and Table 1 in the Supporting Information (SI). The IP value of PTB7 (5.03 $\mathrm{eV}$ ) was indeed virtually identical to that of PCPDTBT (5.00 eV). A series of films was fabricated by spin-coating $\mathrm{P} \mathrm{HT}_{x}$ :PTB7 $1-x$ and $\mathrm{P}_{\mathrm{H}} \mathrm{PT}_{x}$ :PCPDTBT $1-x,(0 \leq x \leq 1)$ from o-DCB solutions, and the morphologies were investigated by atomic force microscopy (AFM) as shown in Figure 1. The tapping mode AFM height images show very distinct blend morphologies for P3HT blended with PTB7 or PCPDTBT. The former shows pronounced phase separation whereas the latter shows no signs of phase separation on length scales that can be resolved by AFM, i.e. a few nm and up. Note that in this case the phase contrast images (not shown) did not yield any additional information, i.e. they are fully consistent with the topography images. In 
particular, at 10\% P3HT in PTB7, the P3HT-rich fraction formed clusters (dark areas in Figure 1b) with sizes varying around $\sim 100-200 \mathrm{~nm}$, distributed in a PTB7-rich matrix, indicative of a spinodal decomposition process. ${ }^{4}$ In contrast, P3HT:PCPDTBT with the same (10\% P3HT) ratio shows a very well mixed morphology (Figure 1e). AFM images covering the full composition range are shown in Figure S1; 2D FFT spectra corresponding to Figure 1 are shown in Figure S2.

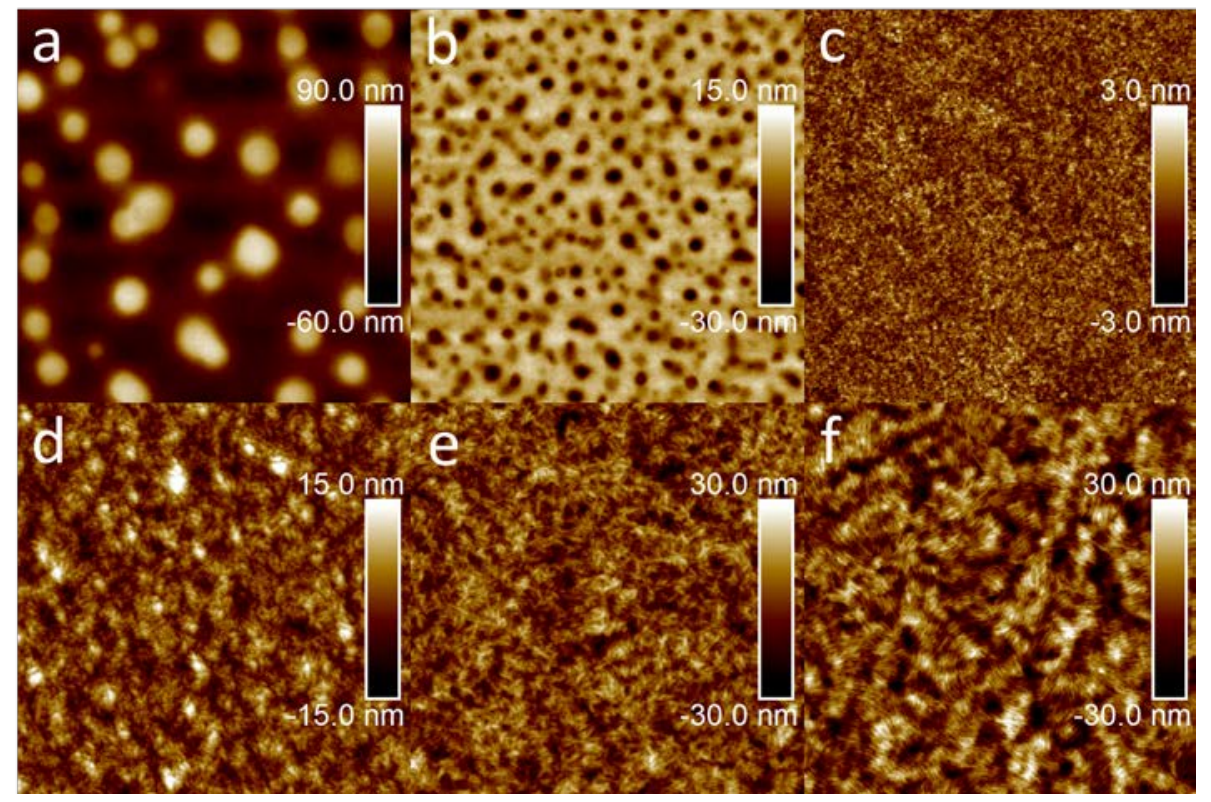

Figure 1. AFM height images for $\mathrm{P}_{3} \mathrm{HT}_{\mathrm{x}}: \mathrm{PTB}_{1-\mathrm{x}}(\mathrm{a}-\mathrm{c})$ and $\mathrm{P} 3 \mathrm{HT} \mathrm{x}$ :PCPDTBT ${ }_{1-\mathrm{x}}(\mathrm{d}-\mathrm{f})$ for $\mathrm{x}=$ $0.6(a, d) ; x=0.1(b, e) ; x=0$ (c, f). All scans are $5 \times 5 \mu \mathrm{m}$.

\subsection{Conductivity and Seebeck coefficient}

The thermoelectric properties of the two blend systems studied above were investigated by measuring the conductivity and Seebeck coefficient on lateral (in-plane) devices of P3HT blended with PTB7 or PCPDTBT. The active layer was p-type doped with $\mathrm{F}_{4} \mathrm{TCNQ}$ using sequential doping, i.e. by spin coating a dopant-containing solution over the previously spin coated blend film, minimizing the perturbation of the morphology. ${ }^{20}$ The results are shown in Figure 2, together with the corresponding power factor $P F=\sigma S^{2}$. Full experimental details are given in the Experimental section. 


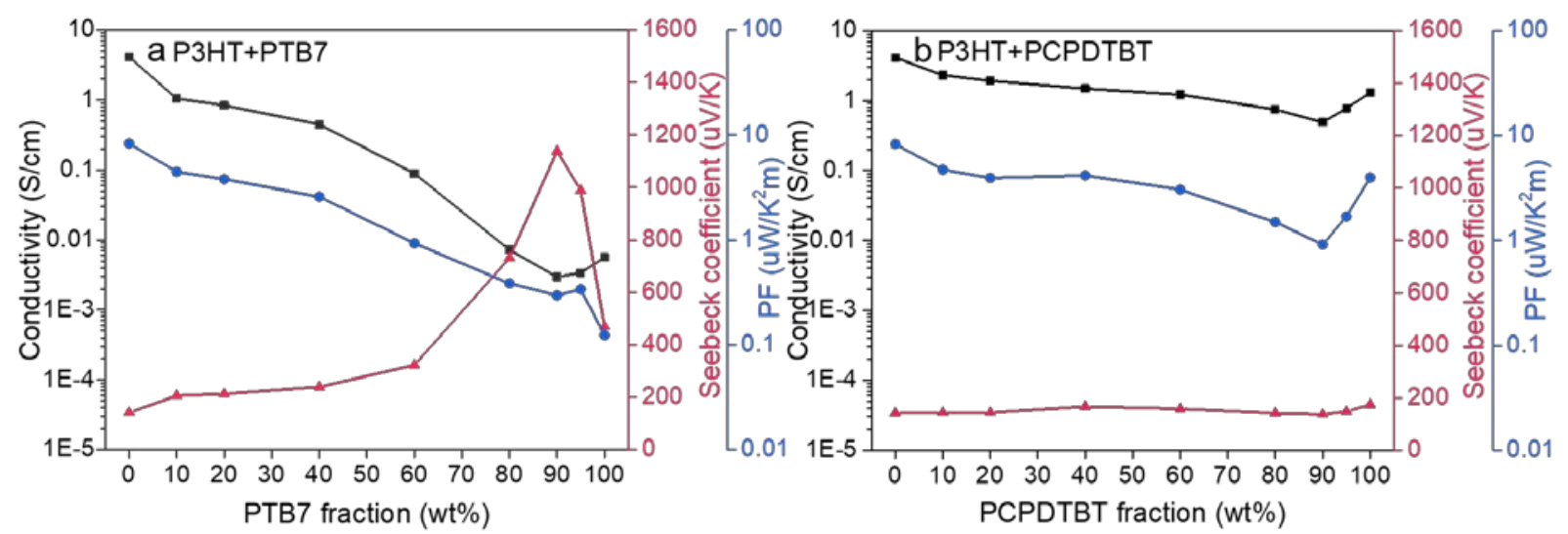

Figure 2. Conductivity, Seebeck coefficient and power factor (PF) for P3HT:PTB7 (a, data taken from our previous work Ref. ${ }^{19}$ ); P3HT:PCPDTBT (b), dependent on active layer composition. The IP of P3HT (4.7 eV) is lower than that of PTB7 $(5.0 \mathrm{eV})$, which leads to a more efficient electron transfer from the HOMO of P3HT to the LUMO of $\mathrm{F}_{4}$ TCNQ (electron affinity $5.24 \mathrm{eV}$, Ref. $^{21}$ ), giving a conductivity of pure P3HT of over $4 \mathrm{~S} / \mathrm{cm}$, whereas a conductivity of pure PTB7 $\sim 6 \times 10^{-3} \mathrm{~S} / \mathrm{cm}$ is found. The corresponding Seebeck coefficients are $S \sim 142$ and 469 $\mu \mathrm{V} / \mathrm{K}$, respectively, consistent with a lower doping level in PTB7. When P3HT is blended with 90\% PTB7, the Seebeck coefficient reaches a peak value of over $1100 \mu \mathrm{V} / \mathrm{K}$, as we demonstrated in a previous work. ${ }^{19}$ In that work, we argued that the reason for the peak is the fact that the small P3HT fraction causes a controlled 'trap' level in the PTB7-dominated density of states (DOS) that pins the Fermi level $E_{F}$ while the transport level $E_{t r}$ remains near the main (PTB7) DOS maximum. This leads to a high Seebeck coefficient as $\mathrm{S}=\left(\mathrm{E}_{\mathrm{F}}-\mathrm{E}_{\mathrm{tr}}\right) / \mathrm{T}$.

Despite having a similar IP, the conductivity of pure PCPDTBT is substantially higher than that of pure PTB7, which we tentatively attribute to a lower disorder and/or a higher attempt-to-hop frequency, which is out of the scope of the present paper. We also note that both blend systems show a slight drop in conductivity when moving away from a single material, which we attribute to the minority compound perturbing the morphology of the pristine majority compound. More 
interestingly, we found that, at the same ratio of 90\% PCPDTBT, no peak in the Seebeck coefficient appeared. In fact, the Seebeck coefficient is almost constant regardless of the P3HT:PCPDTBT blend ratio. This behavior is inconsistent with the predictions of our previous model. ${ }^{19}$ Below, we will show that indeed the reason for the different trend in Seebeck coefficient lies in the morphology and we will extend our model accordingly.

\subsection{Morphology-dependent thermopower}

To explain the impact of morphology on the thermopower in blends of OSC, we propose the mechanism shown in Figure 3. As before, we assume that charge transport in OSC occurs through thermally activated tunneling, i.e. hopping. In contrast to our original work, we will take non-nearest neighbor hopping into account, i.e. we will consider variable range hopping (VRH). According to percolation theory, the conductivity in a VRH system is governed by pathways that optimize the trade-off between hopping to sites that are near in energy (that statistically will not always be spatially nearby) and sites that are spatially near (that statistically will be further away in energy). The parameter quantifying the penalty for hopping over longer distances is the localization radius $\alpha$, according to $p \propto \exp \left(-2 \alpha r_{i f}\right)$ with $p$ the tunneling probability and $r_{i f}$ the distance between initial and final sites. Figure 3 illustrates the site distribution, percolation pathways and DOS with $E_{F}$ and $E_{t r}$ in phase-separated and well-mixed morphologies. Since material A has a HOMO that sits closer to the vacuum level than material B, most mobile charges (holes) and therefore $E_{F}$ will be located in the part of the DOS that corresponds to material A, provided that the charge carrier concentration is less than the concentration of the minority material A. For the present case, the doping method makes an accurate dopant or charge carrier determination hard, but previously we estimated the doping concentration to be around 
$10^{-2},{ }^{19}$ i.e. significantly less than even the smallest concentration of minority material used (10 wt\%); we anticipate this condition to be met for most practical situations.

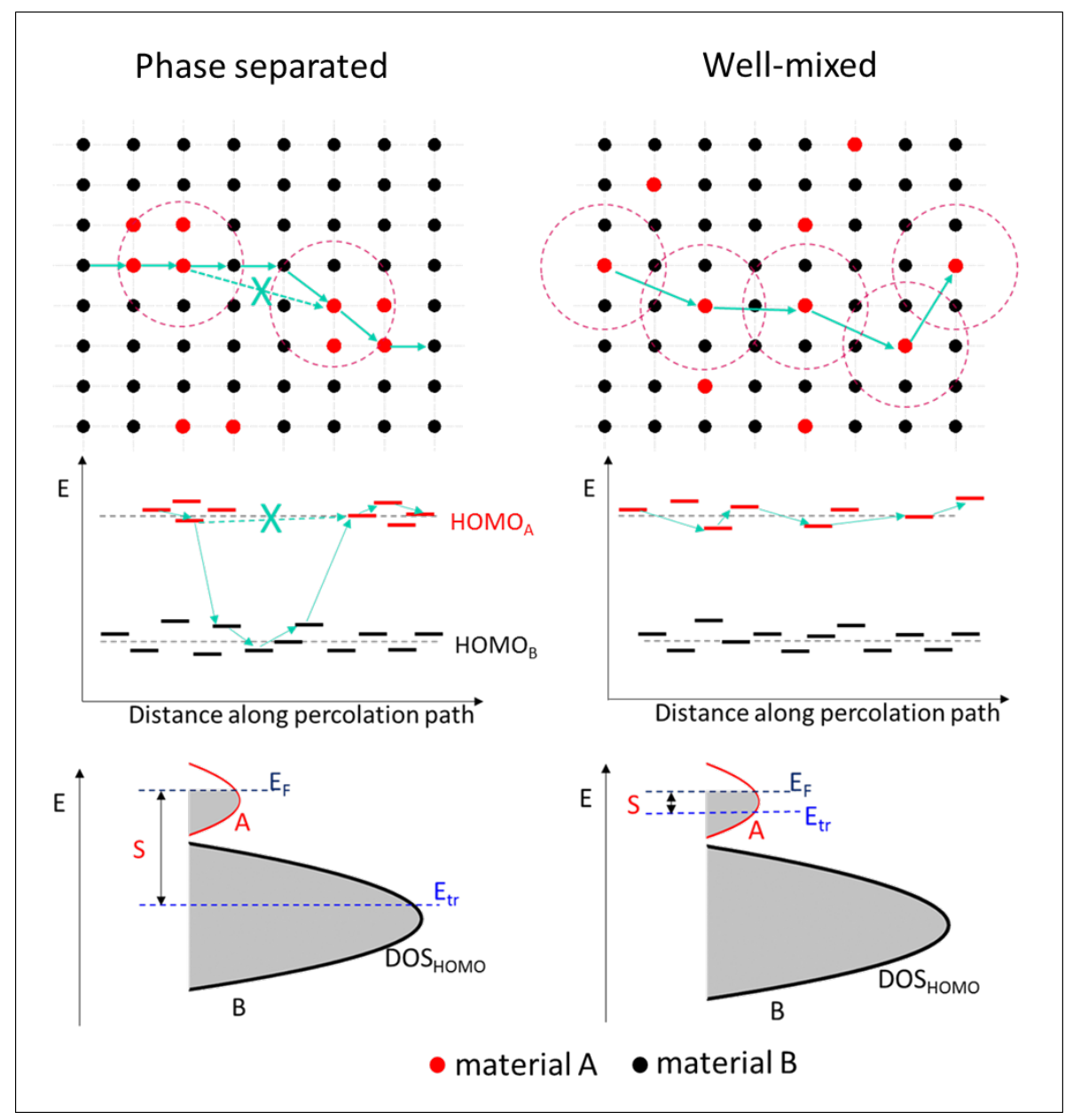

Figure 3. Schematic overview of (top) the site distribution in a 2-component blend (dashed circles indicate the typical hopping distance; green arrows indicate the hopping path); (middle) corresponding energy diagram; (bottom) density of states and characteristic energies, in (left) phase separated and (right) well-mixed morphologies with the same blend ratio. Materials A and B are indicated by red and black colors, respectively.

In case of strong phase separation, material A forms clusters in material B that are far away from each other, i.e. the individual clusters are not within a typical tunneling distance of each other. In Figure 3 (left side) this is indicated by the dashed circle being smaller than the typical 
inter-cluster distance. Hence, the percolating pathway must contain sites from material B, as indicated by the green arrows. This, in turn, causes the transport energy to shift towards the DOS of material B as shown in the left bottom panel. Since the Fermi energy is still located on material A, this leads to a high Seebeck coefficient $S \propto E_{F}-E_{t r}$. For a well-mixed blend morphology, material A sites are randomly distributed in material B. In this case it is possible to form a percolating path that only includes sites from material A since the typical distance between sites of material A is less than the dashed circle in Figure 3 (right side). Therefore, the transport energy and Fermi energy are both located on the DOS of material A, and the Seebeck coefficient remains largely unaffected by the blending with material B. In other words, a measurement of the Seebeck coefficient of a blend material can effectively reveal which sites are involved in the electronic transport. In addition, it can act as an indirect measure of morphology.

\section{4 kinetic Monte Carlo simulations}

To confirm that the mechanism of Figure 3 can indeed explain the morphology dependent behavior of the conductivity and thermopower shown in Figure 2, we carried out numerical kinetic Monte Carlo simulations. The model is similar to the one we used in our previous work Ref. ${ }^{19}$, with the important differences that non-nearest neighbor hops are possible and that (more realistic) random lattices can be (and have been) used. Hopping probabilities are calculated using the Miller-Abrahams rates, with site energies that account for both the typical Gaussian disorder and the full Coulomb interactions with all (static) ionized dopants and all other mobile charges. Sites representing materials A and B only differ in HOMO energy, i.e. all other parameters are taken equal for simplicity. Initially, A and B sites are randomly assigned to lattice sites. Clustered morphologies are generated using the numerical annealing procedure developed by Peumans et al. ${ }^{22,23}$ Typical examples of the used morphologies are shown in Figure 4. 
We should here point out that Figure 3 and its implementation in the numerical model are conceptual only - e.g. the length scales of phase separation in the actual samples of several hundreds of nm, see Figure 1, do not at all match those in the simulations, see Figure 4. The typical length scale in the latter is the mean inter-site distance $a_{N N}$, which is related to the mobility prefactor as $\mu_{0} \propto a_{N N}^{2}$. We do think the concept (and model) are relevant to the actual system as we expect the percolation problem to be largely scale invariant. The quasi-quantitative agreement with experiment of the model results presented below confirms this notion.
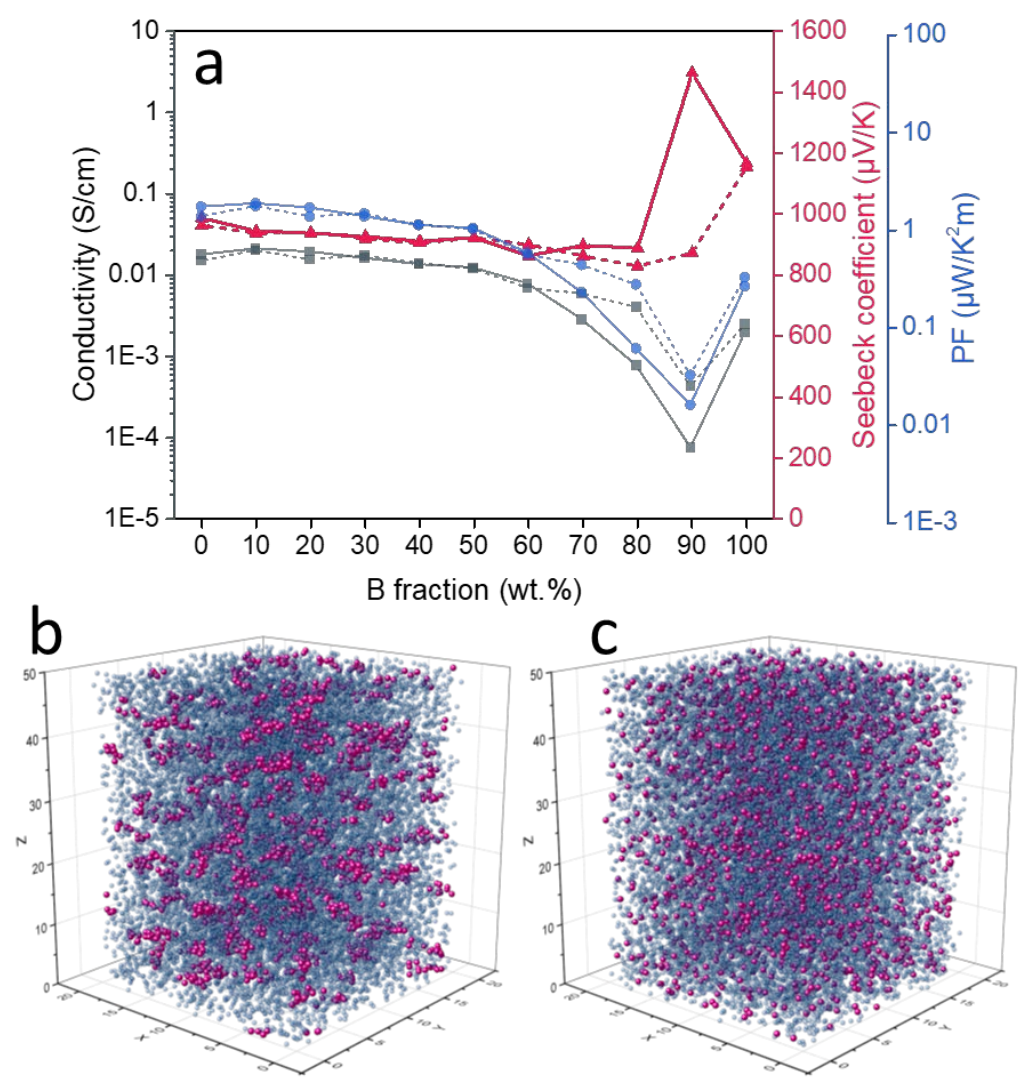

Figure 4. (a) Conductivity, Seebeck coefficient and power factor vs. composition from kinetic Monto Carlo simulations for $\mathrm{A}_{x}: \mathrm{B}_{1-x}$ mixtures with clusters (solid lines) and without clusters (dashed lines). (b) and (c) Typical morphologies used in kinetic Monto Carlo simulations for 
$\mathrm{A}_{10}: \mathrm{B}_{90}$ mixtures $\mathrm{w} /$ and w/o clustering of the A component. Parameters used are the HOMO energy difference $\Delta E_{\text {Номо }}=0.3 \mathrm{eV}$ and doping concentrations $c=10^{-3}$; the attempt to hop frequency $v_{0}=10^{-13} \mathrm{~s}^{-1}$; intersite distance $a_{N N}=1.8 \mathrm{~nm}$; Gaussian disorder $\sigma_{D O S}=0.075 \mathrm{eV}$; temperature $T=300 \mathrm{~K} ; \mathrm{HOMO}_{\mathrm{A}}-\mathrm{LUMO}_{\text {dopant }}=0.24 \mathrm{eV}$; inverse localization radius $\alpha=0.5$ $\mathrm{nm}$; correlation radius of the A compound clusters in units of $a_{N N} r_{C}=1.5$ or 0 .

The trends in conductivity and thermopower that were calculated from the kinetic Monte Carlo model are consistent with those observed experimentally, both for phase separated (P3HT:PTB7) and well-mixed (P3HT:PCPDTBT) morphologies, see Figure 4a. In particular, the distinct behavior of the peak in thermopower for the different morphologies is well reproduced. We attribute the different values of the baseline of the thermopower in experiment ( $S \approx 200 \mu \mathrm{V} / \mathrm{K}$ ) and simulation $(S \approx 900 \mu \mathrm{V} / \mathrm{K})$ to the relatively low $\left(c=10^{-3}\right)$ doping concentration that we used in the latter. For the same reason the simulated conductivity is about 2 orders of magnitude lower than in the experiment. Unfortunately, calculation times become prohibitively large at higher concentrations. These differences notwithstanding, the numerical experiments in Figure 4 confirm the viability of the concept illustrated in Figure 3 and rationalize the experimental results in Figure 2.

\subsection{Controlling thermopower through morphology}

To also experimentally test the proposed relation between morphology and thermoelectric properties, we repeated the conductivity and thermopower measurements on P3HT:PTB7 films to which $5 \%$ DIO by volume was added to the casting solution to suppress phase separation. ${ }^{24,25}$ The AFM height images, shown for $\mathrm{P}_{3} \mathrm{HT}_{0.1}: \mathrm{PTB}_{0.9}$ in Figure 5, indicate that indeed the phase separation is strongly suppressed by the DIO; AFM images at other compositions can be found in SI Figure S3. Importantly, the peak in thermopower vanishes with suppressed phase 
separation while the conductivity increases, see the solid lines in Figure 5a. This finding is consistent with the results of the simulations in Figure 4 and also experimentally confirms our interpretation in terms of morphology of the difference in thermoelectric behavior between the P3HT:PTB7 and P3HT:PCPDTBT systems.

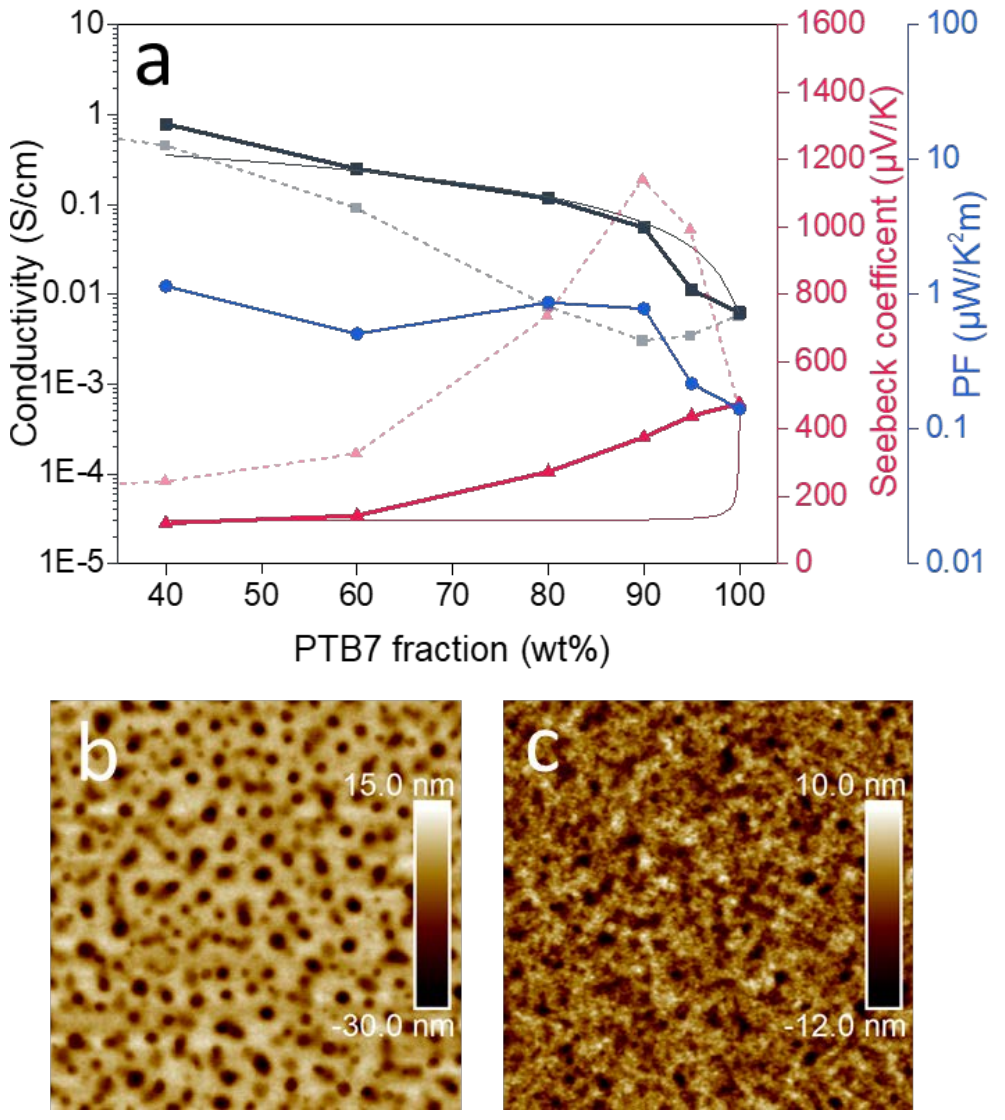

Figure 5. (a) Conductivity, Seebeck coefficient and power factor (PF) for P3HT:PTB7 with 5\% DIO dependent on active layer composition. Dashed lines are data from Figure 2a, i.e. without DIO; thin solid lines indicate an interpolation between binary extremes using an effective medium model. AFM image for $\mathrm{P}_{3} \mathrm{HT}_{0.1}: \mathrm{PTB}_{0.9}$ without DIO (b) and with 5\% DIO (c).

The thin solid lines in Figure 5 show the interpolation between the binary extremes using an effective medium model, viz. and $\mathrm{S}(\mathrm{x})=\left(\mathrm{x} \sigma_{\mathrm{A}} \mathrm{S}_{\mathrm{A}}+(1-\mathrm{x}) \sigma_{\mathrm{B}} \mathrm{S}_{\mathrm{B}}\right) /\left(\mathrm{x} \sigma_{\mathrm{A}}+(1-\mathrm{x}) \sigma_{\mathrm{B}}\right)$, and $\sigma(x)=\mathrm{x} \sigma_{\mathrm{A}}+(1-\mathrm{x}) \sigma_{\mathrm{B}}$, with $x$ the fraction of material B and $S_{A / B}$ and $\sigma_{A / B}$ the values of the 
pure compounds, to highlight the fact that peaked shape of the $S$ vs. composition curves is not a straightforward blending effect. ${ }^{19}$ For the DIO-treated material, $S$ of the blend can be reasonably well approximated by the effective medium model, i.e. a linear combination of the Seebeck coefficients of the constituents weighted by their electrical conductivities. This may seem counterintuitive as pure (or enriched) phases can only be identified in the pristine blend, for which the effective medium scheme evidently fails to reproduce the peak in $S$. The reason for this failure is twofold. First, it seems unlikely that the minority phases in Figures 1a,b and 5b percolate in the in-plane direction, i.e. they do not form a parallel conduction channel as assumed in the expression for $\mathrm{S}(\mathrm{x})$. Second, the effective medium model ignores the electronic interactions in the form of hopping between A and B sites that we have shown to be key in organic thermoelectric blends. ${ }^{19}$

Having shown the consistency of our findings with VRH, we should stress that the absence of an increased thermopower at 5-10\% of material A in Figure 2b, i.e. for $5-10 \%$ P3HT in PCPDTBT, cannot be understood by only considering nearest neighbor hops. In this concentration range only 1 in $10-20$ sites is material A. To form a percolating network that only contains A-sites, each A-site must be connected to at least 2 other A-sites - one bond 'in' and one bond 'out', see Figure 3. Since the number of nearest neighbor sites is $~ 10$ (the coordination number is 6 and 12 for simple cubic and face-centered cubic lattices, respectively) this translates into the demand that at least 1 in $\sim 5$ sites is of the A-type. This is inconsistent with the actual concentration of 1 in 10-20. The implication of this is that also for well-mixed morphologies and variable range hopping, it should be possible to obtain a peak in $S$ at very low concentrations of the 'trapping' compound A, when the inter-(A-)site distance is sufficiently large. We failed to realize this in experiments, which we attribute to the difficulty in actually forming the required 
diluted morphologies and the increasing importance of parallel conduction pathways in the majority material. ${ }^{19}$

\section{Conclusion}

In conclusion, we investigated the conductivity and thermopower of P3HT blended with two other polymers (PTB7 and PCPDTBT) that have a nearly identical ionization potential as measured by UPS. For P3HT:PTB7, the Seebeck coefficient peaked at $\sim 1100 \mu \mathrm{V} / \mathrm{K}$ for $10 \%$ P3HT, whereas no peak was observed for P3HT:PCPDTBT. AFM images showed pronounced phase separation in P3HT:PTB7 blends but not in P3HT:PCPDTBT blends that appeared wellmixed at all ratios. We used a percolation argument to qualitatively understand how the morphology determines the conductivity and the thermopower. The results of explicit numerical simulations using a kinetic Monte Carlo model are in good agreement with the experimental data, confirming the proposed mechanism. In addition, adding 5\% DIO in P3HT:PTB7 to suppress the phase separation makes the peak in $S$ disappeared, corroborating the proposed relation between morphology and thermopower.

We think these findings have a significant relevance beyond the design of high-performance thermoelectrics. They show that in well-mixed binary blends of organic semiconductors, as e.g. used in binary and ternary solar cells, charge transport is dominated by the compound with the lowest LUMO, or highest HOMO for hole transport, even if this compound makes up only a small fraction of all sites; the other compound(s) act merely as an inert matrix, unless phase separation or extreme dilution increases the inter 'trap' distance beyond the (variable) hopping range. $^{26}$ The desired degree of phase separation will depend on the application, with phase separation in pure domains being desired for high-thermopower applications while being undesired for high conductivity. Of course, these rules of thumb break down when phase 
separation occurs on the length scale of the distance between the contacts, as can easily happen in out-of-plane devices like diodes and solar cells.

\section{Experimental Section}

Materials: Regioregular-poly(3-hexylthiophene-2,5-diyl) (rr-P3HT), Poly(\{4,8-bis[(2ethylhexyl)oxy]benzo[1,2-b:4,5-b’]dithiophene-2,6-diyl\}\{3-fluoro-2-[(2-ethylhexyl) carbonyl]thieno[3,4-b]thiophenediyl\}) (PTB7), and Poly[2,6-(4,4-bis-(2-ethylhexyl)-4Hcyclopenta [2,1-b;3,4-b’]dithiophene)-alt-4,7(2,1,3-benzothiadiazole)] (PCPDTBT) were purchased from 1-Material Inc. 2,3,5,6-Tetrafluoro-7,7,8,8-tetracyanoquinodimethane ( $\mathrm{F}_{4} \mathrm{TCNQ}$ ) was purchased from Ossila.

Device fabrication: P3HT, PTB7, and PCPDTBT were dissolved in ortho-dichlorobenzene (oDCB), respectively, to make solutions of $25 \mathrm{mg} / \mathrm{ml}$. $\mathrm{F}_{4} \mathrm{TCNQ}$ was dissolved in a mixture of tetrahydrofuran (THF) and dichloromethane (DCM) in a 4:1 ratio at a concentration of $5 \mathrm{mg} / \mathrm{ml}$. P3HT and PTB7 (or PCPDTBT) solutions were mixed by volume to get the desired blend ratio (from $0 \%$ to $100 \%$ in steps of $10 \%$ ). For some P3HT:PTB7 solutions 5\% DIO by volume was added to suppress phase separation. The active layers were spin-coated (1000 rpm for 60 seconds and then 3000 rpm for 20 seconds) on top of cleaned glass substrates to get a thickness around $150 \mathrm{~nm}$ as measured with a Dektak surface profilometer. $\mathrm{F}_{4} \mathrm{TCNQ}$ was spin-coated on top of the active layer at $3000 \mathrm{rpm}$ for 40 seconds. After that the $\mathrm{MoO}_{3}(9 \mathrm{~nm}) / \mathrm{Al}(90 \mathrm{~nm})$ contacts were evaporated through a shadow mask under a pressure of $1 \times 10^{-6} \mathrm{mbar}$.

Conductivity and Seebeck coefficient measurement: Room temperature electrical characterizations were performed in a glove box under dry $\mathrm{N}_{2}$ atmosphere. Current-voltage characteristics were obtained between $-50 \mathrm{mV}$ and $50 \mathrm{mV}$ and conductivities calculated according 
to $\sigma=J / F$, where $J$ and $F$ are the current density and electric field, respectively. The thermopower was obtained by applying a linear temperature gradient of various magnitudes $\Delta T$ along the sample and recording the shift of the JV characteristics at the end of a settling time of ca. 400 seconds. From the change of the thermovoltage $\Delta V$ as a function of $\Delta T$ the thermopower can be calculated as $\Delta V=S \Delta T$. In general, all electrical measurements have been done in a Kelvin-probe (4-probe) geometry.

AFM measurement: AFM images were taken using a Veeco Dimension 3100 AFM in tapping (intermittent contact) mode in air using a Nanosensors NCHR tip with a nominal apex radius $<10 \mathrm{~nm}$.

UPS measurement: Ultraviolet photoemission spectroscopy (UPS) experiments were carried out using a Scienta ESCA 200 spectrometer in ultrahigh vacuum $\left(1 \times 10^{-10}\right.$ mbar $)$ with a standard He-discharge lamp with HeI $21.22 \mathrm{eV}$. The total energy resolution of the UPS measurement is about $80 \mathrm{meV}$ as extracted from the width of the Fermi level of clean gold foil. All spectra were collected at a photoelectron takeoff angle of $0^{\circ}$ (normal emission). The work functions of the films were extracted from the determination of the high binding-energy cutoff of the UPS spectra by applying a bias of $-3 \mathrm{~V}$ to the sample.

\section{ASSOCIATED CONTENT}

Supporting Information. A listing of the contents of each file supplied as Supporting Information should be included.

AFM height images for P3HT:PTB7 and P3HT:PCPDTBT with full ratio; UPS spectra of pristine of P3HT, PTB7, and PCPDTBT; AFM height images for PEHT:PTB7 with 5 v. \% DIO; Fermi energy and ionization potential of P3HT, PTB7, and PCPDTBT. (PDF) 
AUTHOR INFORMATION

Corresponding Author

*Tel: +46 700895377; Fax: -; E-mail: martijn.kemerink@liu.se

\section{Author Contributions}

\section{Funding Sources}

Any funds used to support the research of the manuscript should be placed here (per journal style).

Notes

Any additional relevant notes should be placed here.

\section{ACKNOWLEDGMENT}

The research by G.Z. is supported by the China Scholarship Council (CSC). We are grateful to Hassan Abdallah for technical assistance with the thermoelectric measurements, and Olle Inganäs for the use of technical infrastructure.

\section{REFERENCES}

(1) Lee, J. K.; Ma, W. L.; Brabec, C. J.; Yuen, J.; Moon, J. S.; Kim, J. Y.; Lee, K.; Bazan, G. C.; Heeger, A. J. Processing Additives for Improved Efficiency from Bulk Heterojunction Solar Cells. J. Am. Chem. Soc. 2008, 130 (11), 3619-3623. 
(2) Park, S. H.; Roy, A.; Beaupré, S.; Cho, S.; Coates, N.; Moon, J. S.; Moses, D.; Leclerc, M.; Lee, K.; Heeger, A. J. Bulk Heterojunction Solar Cells with Internal Quantum Efficiency Approaching 100\%. Nat. Photonics 2009, 3 (5), nphoton.2009.69.

(3) Liu, Y.; Zhao, J.; Li, Z.; Mu, C.; Ma, W.; Hu, H.; Jiang, K.; Lin, H.; Ade, H.; Yan, H. Aggregation and Morphology Control Enables Multiple Cases of High-Efficiency Polymer Solar Cells. Nat. Commun. 2014, 5, ncomms6293.

(4) Kouijzer, S.; Michels, J. J.; van den Berg, M.; Gevaerts, V. S.; Turbiez, M.; Wienk, M. M.; Janssen, R. A. J. Predicting Morphologies of Solution Processed Polymer:Fullerene Blends. J. Am. Chem. Soc. 2013, 135 (32), 12057-12067.

(5) Franeker, J. J. van; Turbiez, M.; Li, W.; Wienk, M. M.; Janssen, R. A. J. A Real-Time Study of the Benefits of Co-Solvents in Polymer Solar Cell Processing. Nat. Commun. 2015, 6, ncomms7229.

(6) V. Mikhnenko, O.; Azimi, H.; Scharber, M.; Morana, M.; M. Blom, P. W.;

Antonietta Loi, M. Exciton Diffusion Length in Narrow Bandgap Polymers. Energy Environ. Sci. 2012, 5 (5), 6960-6965.

(7) Patel, S. N.; Glaudell, A. M.; Peterson, K. A.; Thomas, E. M.; O’Hara, K. A.; Lim, E.; Chabinyc, M. L. Morphology Controls the Thermoelectric Power Factor of a Doped Semiconducting Polymer. Sci. Adv. 2017, 3 (6), e1700434.

(8) Hamidi-Sakr, A.; Biniek, L.; Bantignies, J.-L.; Maurin, D.; Herrmann, L.; Leclerc, N.; Lévêque, P.; Vijayakumar, V.; Zimmermann, N.; Brinkmann, M. A Versatile Method to Fabricate Highly In-Plane Aligned Conducting Polymer Films with Anisotropic Charge Transport and Thermoelectric Properties: The Key Role of Alkyl Side Chain Layers on the Doping Mechanism. Adv. Funct. Mater. 2017, 27 (25), 1700173. 
(9) Russ, B.; Glaudell, A.; Urban, J. J.; Chabinyc, M. L.; Segalman, R. A. Organic

Thermoelectric Materials for Energy Harvesting and Temperature Control. Nat. Rev. Mater. 2016, 1 (10), natrevmats201650.

(10) van Reenen, S.; Scheepers, M.; van de Ruit, K.; Bollen, D.; Kemerink, M. Explaining the Effects of Processing on the Electrical Properties of PEDOT:PSS. Org. Electron. 2014, 15 (12), 3710-3714.

(11) Weathers, A.; Khan, Z. U.; Brooke, R.; Evans, D.; Pettes, M. T.; Andreasen, J. W.; Crispin, X.; Shi, L. Significant Electronic Thermal Transport in the Conducting Polymer Poly(3,4-Ethylenedioxythiophene). Adv. Mater. 2015, 27 (12), 2101-2106.

(12) Shi, W.; Zhao, T.; Xi, J.; Wang, D.; Shuai, Z. Unravelling Doping Effects on PEDOT at the Molecular Level: From Geometry to Thermoelectric Transport Properties. J. Am. Chem. Soc. 2015, 137 (40), 12929-12938.

(13) Ouyang, L.; Musumeci, C.; Jafari, M. J.; Ederth, T.; Inganäs, O. Imaging the Phase Separation Between PEDOT and Polyelectrolytes During Processing of Highly Conductive PEDOT:PSS Films. ACS Appl. Mater. Interfaces 2015, 7 (35), 19764-19773.

(14) Crispin, X.; Jakobsson, F. L. E.; Crispin, A.; Grim, P. C. M.; Andersson, P.; Volodin, A.; van Haesendonck, C.; Van der Auweraer, M.; Salaneck, W. R.; Berggren, M. The Origin of the High Conductivity of Poly(3,4-Ethylenedioxythiophene)-Poly(Styrenesulfonate) (PEDOT-PSS) Plastic Electrodes. Chem. Mater. 2006, 18 (18), 4354-4360.

(15) Bubnova, O.; Khan, Z. U.; Malti, A.; Braun, S.; Fahlman, M.; Berggren, M.; Crispin, X. Optimization of the Thermoelectric Figure of Merit in the Conducting Polymer Poly(3,4Ethylenedioxythiophene). Nat. Mater. 2011, 10 (6), 429-433. 
(16) Saxena, N.; Čorić, M.; Greppmair, A.; Wernecke, J.; Pflüger, M.; Krumrey, M.; Brandt, M. S.; Herzig, E. M.; Müller-Buschbaum, P. Morphology-Function Relationship of Thermoelectric Nanocomposite Films from PEDOT:PSS with Silicon Nanoparticles. Adv. Electron. Mater. 2017, 3 (8), 1700181.

(17) Lu, G.; Bu, L.; Li, S.; Yang, X. Bulk Interpenetration Network of Thermoelectric Polymer in Insulating Supporting Matrix. Adv. Mater. 2014, 26 (15), 2359-2364.

(18) Abbaszadeh, D.; Kunz, A.; Wetzelaer, G. a. H.; Michels, J. J.; Crăciun, N. I.; Koynov, K.; Lieberwirth, I.; Blom, P. W. M. Elimination of Charge Carrier Trapping in Diluted Semiconductors. Nat. Mater. 2016, 15 (6), 628-633.

(19) Zuo, G.; Liu, X.; Fahlman, M.; Kemerink, M. High Seebeck Coefficient in Mixtures of Conjugated Polymers. Adv. Funct. Mater. 2017, 1703280.

(20) Scholes, D. T.; Hawks, S. A.; Yee, P. Y.; Wu, H.; Lindemuth, J. R.; Tolbert, S. H.;

Schwartz, B. J. Overcoming Film Quality Issues for Conjugated Polymers Doped with F4TCNQ by Solution Sequential Processing: Hall Effect, Structural, and Optical Measurements. J. Phys. Chem. Lett. 2015, 6 (23), 4786-4793.

(21) Gao, W.; Kahn, A. Controlled P-Doping of Zinc Phthalocyanine by Coevaporation with Tetrafluorotetracyanoquinodimethane: A Direct and Inverse Photoemission Study. Appl. Phys. Lett. 2001, 79 (24), 4040-4042.

(22) Peumans, P.; Uchida, S.; Forrest, S. R. Efficient Bulk Heterojunction Photovoltaic Cells Using Small-Molecular-Weight Organic Thin Films. Nature 2003, 425 (6954), nature01949.

(23) Marsh, R. A.; Groves, C.; Greenham, N. C. A Microscopic Model for the Behavior of Nanostructured Organic Photovoltaic Devices. J. Appl. Phys. 2007, 101 (8), 083509. 
(24) Lou, S. J.; Szarko, J. M.; Xu, T.; Yu, L.; Marks, T. J.; Chen, L. X. Effects of Additives on the Morphology of Solution Phase Aggregates Formed by Active Layer Components of HighEfficiency Organic Solar Cells. J. Am. Chem. Soc. 2011, 133 (51), 20661-20663.

(25) Chen, Y.; Zhang, S.; Wu, Y.; Hou, J. Molecular Design and Morphology Control Towards Efficient Polymer Solar Cells Processed Using Non-Aromatic and Non-Chlorinated Solvents. Adv. Mater. 2014, 26 (17), 2744-2749.

(26) Melianas, A.; Pranculis, V.; Spoltore, D.; Benduhn, J.; Inganäs, O.; Gulbinas, V.; Vandewal, K.; Kemerink, M. Charge Transport in Pure and Mixed Phases in Organic Solar Cells. Adv. Energy Mater. 2016, 6 (18), 1600699. 
Table of Contents Graphic

Well-mixed Phase separated

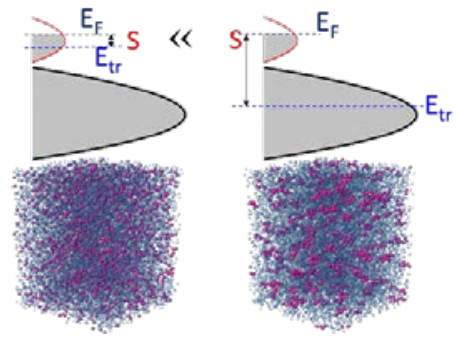

